\section{Neural Bases of Human Working Memory}

\author{
Edward E. Smith ${ }^{1}$ \\ Department of Psychology, University of Michigan, Ann Arbor, Michigan
}

These results indicated that human spatial working memory is partly mediated by regions in parietal and prefrontal cortex. Subsequent research has shown that a different neural system is involved when people store object (rather than spatial) information, a difference similar to that found in monkeys.

\section{Keywords}

working memory; spatial working memory; neuroimaging; prefrontal cortex

Psychologists have long been concerned with human working memory, the kind of memory that allows us to maintain information briefly in an active state. Sometimes we keep information active until we externalize it, as when we hold onto a phone number until we dial it; other times we keep information active so we can operate on it, as when we store the outcomes of certain calculations while doing an arithmetic problem "in our heads" (e.g., Baddeley, 1992). Most of the research on working memory has been carried out at the cognitive level and has described working memory in terms of its functional properties. Recently, though, researchers have been able to study working memory at the neural level as well as the cognitive one, and this neurally based work is the focus of the present article.

\section{WORKING MEMORY IN MONKEYS}

A useful starting point is research on working memory in monkeys. In a set of influential experiments, rhesus monkeys were trained to store spatial information for a brief time period. In one study (Funahashi, Bruce, \& Goldman- 
Rakic, 1989), the following sequence of events occurred on each trial: First, a small target square was presented briefly at one of eight random locations. Second, the square disappeared, and the monkey had to remember its position during a 2.5-s delay period. Finally, at the end of the delay period, the monkey was cued to shift its gaze to the position that the square had occupied (and was rewarded for doing so). While the monkey was performing this task, the experimenters recorded activity of individual neurons in various regions of the brain, including the frontal cortex (i.e., the most anterior of the four lobes into which the cortex is divided). These single-cell recordings showed two major findings:

- There were cells in a particular region of the frontal cortexcalled the prefrontal cortexthat were active only during the delay period, when the animal was storing the position of the target square; presumably, these neurons mediated the memory of the target location.

- The neurons in question coded relatively specific locations. That is, there were neurons that had a high firing rate when the target appeared in a specific location, but whose activity did not exceed baseline levels when the target appeared in other spatial locations. These neurons, then, carried the memory of the target locations. Other single-cell recording studies have found such memory cells in parietal cortex (another one of the four lobes of the cortex) as well as frontal cortex (see Fuster, 1997).

The next major development was a demonstration that when the task required storage of object rather than spatial information, different cells in the prefrontal cortex were involved. In an experiment using a paradigm similar to the one just described (Wilson, O'Scalaidhe, \& Goldman-Rakic, 1993), monkeys were required to briefly store either the location of a target square or a nonspatial object such as a visual pattern or a monkey's face; the former task requires spatial working memory, the latter necessitates object working memory. The spatial task led to increased neuronal activation in the same region of prefrontal cortex as found before, but the object task resulted in heightened neural activity in a region of prefrontal cortex that was just below (or ventral to) the spatial region. The object region contains cells that fire only during the delay period and code specific parts or features of objects. The upshot of this research is that our concept of "memory," which has traditionally been somewhat abstract and characterized in terms of functions, has now been given a concrete realization in terms of neurons.

\section{SPATIAL WORKING MEMORY IN HUMANS}

The striking results with monkeys led to an effort to find the neural bases of comparable working memory systems in humans. One cannot record from individual neurons in humans, so the research with humans has used functional neuroimaging, including both positron emission tomography (PET) and functional magnetic resonance imaging (fMRI). Both of these are brain-scanning methods that allow one to determine which neural regions are active when participants perform various tasks, thereby allowing one to infer which neural regions are mediating which cognitive processes. In research on working memory, a standard strategy has been to image participants while they perform a working memory task, and also while they perform a control task that presumably involves the same processes as the memory task except for the memory component. According to this subtraction strategy, the difference between the results for the two tasks reflects only memory processes (Posner, Petersen, Fox, \& Raichle, 1988). ${ }^{2}$

The earliest neuroimaging studies focused on spatial working memory. The spatial memory task used in one experiment (Jonides et al., 1993) was based on the paradigms used in the single-cell research described earlier. The sequence of events on each trial of the human task was as follows: First, three randomly selected dots were presented. Second, the dots disappeared, and the participants had to remember the locations during a 3-s delay period. Finally, at the end of the delay period, a single outline circle (the location probe) was presented, and participants had to decide whether or not it encircled the location of one of the target dots. This task clearly involves spatial working memory, but it also includes other processes-such as encoding spatial information and selecting and executing a response. To determine the effects of these nonstorage processes on the images, Jonides et al. devised a control task that included these processes but not storage. In this task, the three dots were again presented briefly, but they remained in view while the probe circle was presented. Again, participants decided whether the probe encircled one of the target locations, but their decisions could be made perceptually without involving memory.

Participants had their brains scanned by PET while they performed blocks of trials of the spatial working memory task and blocks of trials of the control task. In PET studies, each task is associ- 
ated with a pattern of brain activation, which indirectly reflects the activation of large groups of neurons. In accordance with the subtraction strategy, the results for the control task were subtracted from the results for the memory task to provide an estimate of the neural areas that mediate spatial working memory. This subtraction revealed activations in a number of righthemisphere regions, including the extrastriate occipital cortex, the posterior parietal cortex, and the premotor region of the frontal cortex. These regions are presented schematically in Figure 1.

How well do these activations line up with the single-cell results obtained with monkeys? (Such a question is meaningful because prior work has established detailed correspondences between human brains and the brains of rhesus monkeys.) Recall that the monkey results, like the human findings, revealed heightened neural activation in parietal and frontal cortex.
But there are some discrepancies between the two sets of data. One is that only in humans does spatial memory appear to be specialized, or lateralized, to the right hemisphere, which fits with the tendency for the human brain to show more lateralization of function than do the brains of other primates (e.g., Kolb \& Whishaw, 1996). The second difference between the monkey and human data involves activations in the frontal cortex. In the monkey, spatial memory appears to be mediated by a specific region in the dorsolateral prefrontal cortex, whereas in humans the relevant frontal regions are superior (higher) and inferior (lower) to this. Other early neuroimaging studies of spatial working memory in humans, however, obtained frontal activations that were more in line with the monkey research (e.g., McCarthy et al., 1994). Given this variability in results, it is helpful to consider more recent neuroimaging data.

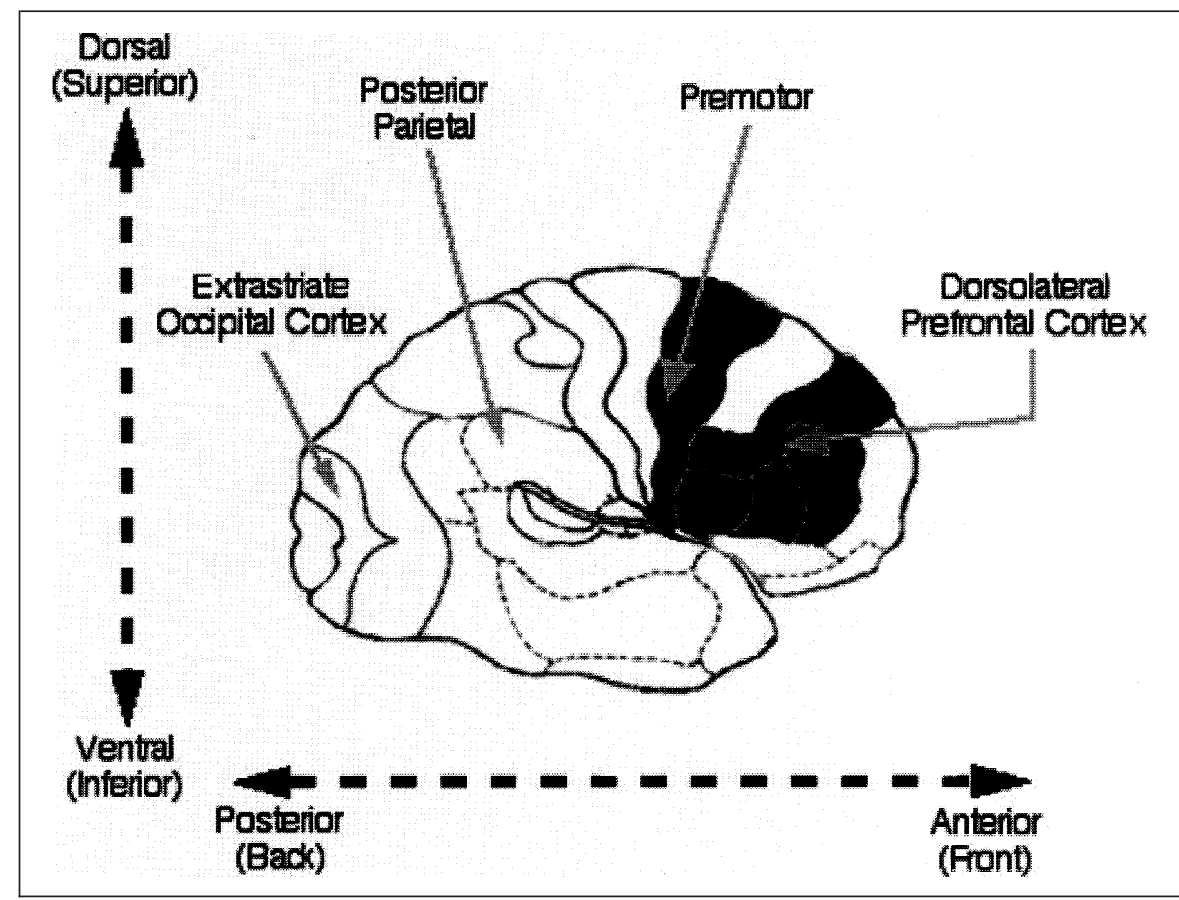

Fig. 1. Schematic of the right side of the cortex, displaying the areas of greatest interest within the frontal, parietal, and occipital lobes. The premotor region and all areas that lie in front of it make up the prefrontal cortex. Also shown are the anteriorposterior and dorsal-ventral directions, which are used in anatomical descriptions.

\section{SPATIAL VERSUS OBJECT WORKING MEMORY IN HUMANS}

Recall that in the single-cell studies with rhesus monkeys, one of the critical findings was that spatial and object working memory were mediated by different regions of the prefrontal cortex. Are spatial and object working memory mediated by different neural systems in humans as well? And if so, how do the different neural circuits correspond to those found in monkeys?

A number of functional neuroimaging experiments have addressed these questions. Several of these studies used an approach in which the same set of visual forms was presented for the spatial and object memory tasks, and all that varied was whether participants were required to remember the positions of the forms (spatial working memory) or the identity of the forms (object working memory). In one illustrative fMRI study, three target faces were presented sequentially in three different positions, followed by a probe face that could appear in any of several positions. In the spatial task, participants decided whether the probe matched any of the targets in position; in the object task, they decided whether the probe matched any of the targets in identity. The spatial task activated a region in right-hemisphere premotor cortex (one of the frontal regions reported in Jonides et al., 1993); in contrast, the object task activated regions in right-hemisphere dorsolateral prefrontal cortex. Thus, spatial and object working memory seem to recruit different neural circuits in humans, as they do in monkeys (Courtney, Ungerleider, Keil, \& Haxby, 1997).

How do the human activation sites line up with those in the monkey? In humans, the premotor area is associated with spatial storage 
and the dorsolateral area with object storage, whereas in monkeys the dorsolateral area is associated with spatial storage. But there is a broader point of agreement between the two sets of studies. For humans as well as monkeys, the frontal regions activated during spatial storage are higher, or more dorsal, than the regions activated during object storage.

The latter conclusion is strengthened by a recent meta-analysis of several functional neuroimaging studies that compared spatial and object working memory in humans (Smith \& Jonides, 1999). The outcome is presented in Figure 2, with black symbols designating activations in spatial memory tasks and gray symbols designating activations in object memory tasks. There is a notable dorsal-ventral difference between spatial and object working memory tasks, with the spatial activations being higher in the cortex, including the prefrontal cortex. The upshot, then, is that the prefrontal cortex is at least partly organized by the kind of material

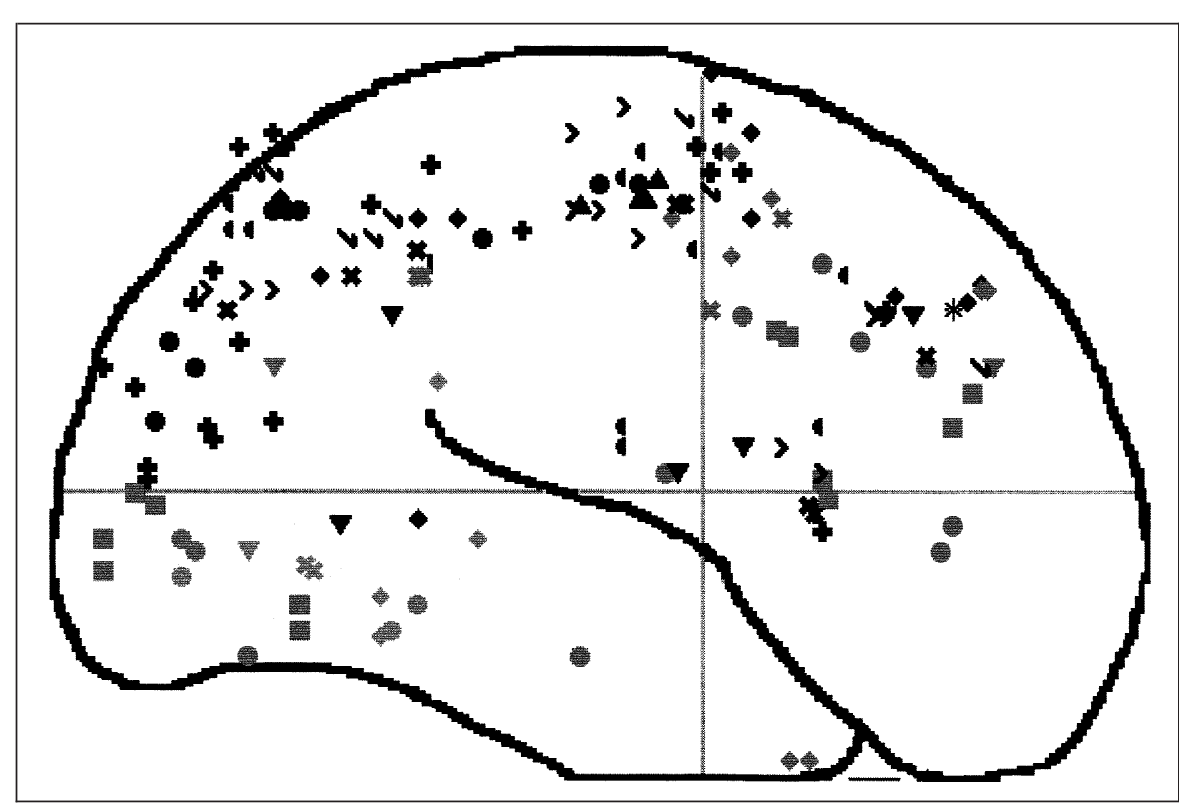

Fig. 2. Schematic of the right side of the cortex, showing regions of activation for spatial (black symbols) and object (gray symbols) working memory tasks in humans (adapted from Smith \& Jonides, 1999). The results are drawn from 11 different experiments, with different symbols being used for different experiments. Not all the activations occurred on the surface of the brain, so the schematic should be interpreted as a glass brain-as though one were looking through the brain from the side. ing memory involves selectively attending to a representation of that location (and recomputing its spatial coordinates). This notion of rehearsal receives support from a comparison of neuroimaging results obtained in studies of spatial working memory and in studies of selective attention (Awh \& Jonides, 1997). The premotor area that is consistently activated in spatial working memory tasks is also routinely activated in tasks involving selective attention. This region, then, may be involved in spatial rehearsal. In contrast, two regions that are activated in spatial working memory tasks but not in spatial attention tasks are the posterior parietal region and the occipital area. These regions may mediate the passive storage component of spatial working memory.

\section{CONCLUSION}

The memory studies with rhesus monkeys have provided a useful model for the study of spatial and object working memory in humans. Using neuroimaging techniques to determine what neural areas are active during task performance, researchers have established that spatial and object working memory have different neural bases in humans. The regions most involved in spatial working memory tend to be dorsal to those involved in object working memory. In particular, the right-hemisphere premotor region and posterior parietal region are consistently activated in studies of spatial but not object working memory, whereas more ventral regions are activated in studies of object working memory. Furthermore, there is suggestive evidence that some of the spatial areas-particularly the premotor one-are mediating the process of rehearsal.

Although this is a promising 
start, we have only begun to understand the neural bases of human working memory. At a global level, we do not know how many different working memory systems there are, nor how they interact with one another. In addition to spatial and object systems, there is evidence for a verbal working memory (e.g., Smith \& Jonides, 1999), but what about other modalities? Are there, for example, separate systems for auditory and tactual information, or for motor and semantic information? At a more detailed level, we do not understand how the spatial and object systems are implemented by neurons. For example, we have evidence that spatial working memory has separate neural components for passive storage and rehearsal, with the righthemisphere premotor area being part of the rehearsal circuit. But this premotor area contains thousands and thousands of neurons. How are these neurons organized so as to implement the function of selectively attending to a stored position? Questions like this are illustrative of the next level of analysis in the study of human working memory.

\section{Recommended Reading \\ Goldman-Rakic, P.S. (1987). Cir- cuitry of primate prefrontal cor- tex and regulation of behavior by}

representational memory. In $\mathrm{F}$. Plum (Ed.), Handbook of physiology, Section 1, Vol. 7 (pp. 373-417). Bethesda, MD: American Physiological Society.

Jonides, J. (1995). Working memory and thinking. In E.E. Smith \& D. Osherson (Eds.), Invitation to cognitive science: Vol. 3. Thinking (2nd ed., pp. 215-265). Cambridge, MA: MIT Press.

Smith, E.E., \& Jonides, J. (1999). (See References)

Acknowledgments-Some of the research reported here was supported by grants from the McDonnell-Pew Program in Cognitive Neuroscience, the National Institute on Aging, and the Office of $\mathrm{Na}$ val Research.

\section{Notes}

1. Address correspondence to Edward E. Smith, Department of Psychology, University of Michigan, 525 East University, Ann Arbor, MI 48109; email: eesmith@umich.edu.

2. Using PET, one determines which neural regions are active by monitoring the flow of a radioactive tracer that has been injected into a participant. Because changes in regional neural activity are known to be accompanied by changes in the blood flow to that region, the measured changes in regional blood flow indicate regions of changed neural activity. With regard to fMRI measurements, the logic is more complex. When increased neural activity leads to an increase in blood flow to a region, not all the increased oxygen brought by the blood to this region is used, leading to a local increase in the amount of oxygenated hemoglobin and a decrease in the amount of deoxygenated hemoglobin. Changes in deoxygenated hemoglobin can affect the magnetic field inherent in the brain, which affects the fMRI signal.

\section{References}

Awh, E., \& Jonides, J. (1997). Spatial selective attention and spatial working memory. In R. Parasurama (Ed.), The attentive brain (pp. 353380). Cambridge, MA: MIT Press.

Baddeley, A. (1992). Working memory. Science, 255, 556-559.

Courtney, S.M., Ungerleider, L.G., Keil, K., \& Haxby, J.V. (1997). Transient and sustained activity in a distributed neural system for human working memory. Nature, 386, 608-611.

Funahashi, S., Bruce, C.J., \& Goldman-Rakic, P.S (1989). Mnemonic coding of visual space in the monkey's dorsolateral prefrontal cortex. Journal of Neurophysiology, 61, 331-349.

Fuster, J.M. (1997). The prefrontal cortex: Anatomy, physiology, and neuropsychology of the frontal lobe. New York: Lippincott-Raven.

Jonides, J., Smith, E.E., Koeppe, R.A., Awh, E., Minoshima, S., \& Mintun, M.A. (1993). Spatial working memory in humans as revealed by PET. Nature, 363, 623-625.

Kolb, B., \& Whishaw, I.Q. (1996). Fundamentals of human neuropsychology (4th ed.). New York: W.H. Freeman.

McCarthy, G.B., Blamire, A.M., Puce, A., Nobre, A.C., Bloch, G., Hyder, F., Goldman-Rakic, P. \& Shulman, R.G. (1994). Functional magnetic resonance imaging of human prefrontal cortex activation during a spatial working memory task. Proceedings of the National Academy of Sciences, USA, 91, 8690-8694.

Posner, M.I., Petersen, S.E., Fox, P.T., \& Raichle, M.E. (1988). Localization of cognitive operations in the human brain. Science, 240, 16271631.

Smith, E.E., \& Jonides, J. (1999). Storage and executive processes in the frontal lobes. Science, 283, 1657-1661.

Wilson, F.A., O'Scalaidhe, S.P. \& Goldman-Rakic P.S. (1993). Dissociation of object and spatial processing domains in primate prefrontal cortex. Science, 260, 1955-1958. 